\title{
Conhecimento e atitudes sobre alimentos irradiados de nutricionistas que atuam na docência
}

\author{
Knowledge and views of professors of nutrition about food irradiation \\ Kelly Daiane SILVA ${ }^{1}$, Vilma de Oliveira BRAGA ${ }^{1}$, Késia Diego QUINTAES ${ }^{1 \star}$, \\ Niurka Maritza Almeyda HAJ-ISA², Eloísa Santos NASCIMENTO³
}

\section{Resumo}

A irradiação de alimentos é eficiente tecnologia que pode ser empregada na conservação dos alimentos. Porém, o conhecimento dos consumidores sobre os alimentos irradiados tem se mostrado insuficiente, resultando em baixa aceitação destes alimentos. Considerando que o nutricionista é o profissional de saúde habilitado para orientar pacientes e consumidores sobre a ingestão e seleção de alimentos, este estudo avaliou o conhecimento e a atitude frente aos alimentos irradiados entre nutricionistas que atuam na docência em instituições de ensino superior em Belo Horizonte - MG, Brasil. Dos 66 participantes, 86,4\% referiram saber o que são alimentos irradiados, todavia $71,2 \%$ desconhecem o processo, $75,8 \%$ desconhecem a legislação, $21,2 \%$ desconhecem as finalidades da irradiação, $12,1 \%$ consideram os alimentos irradiados como sendo radioativos e, 31,8\% acreditam que a irradiação de alimentos resulta em redução do valor nutritivo dos alimentos. Os alimentos irradiados não seriam recusados pelos doutores, porém seriam recusados por 5 mestres e por 6 especialistas. Foi concluído que a formação dos futuros nutricionistas pode estar sendo realizada sem o mínimo de conhecimento necessário a respeito dos alimentos irradiados, podendo estar corroborando para a atitude negativa dos consumidores.

Palavras-chave: ensino; segurança alimentar e nutricional; saúde pública.

\begin{abstract}
Food irradiation is an efficient technology that can be used in the conservation of foods. However, consumers' knowledge about irradiated foods has proved insufficient resulting in low acceptance of such foods. Considering that dietitians and nutritionists are the qualified health professionals to guide patients and consumers towards the ingestion and selection of foods, this study aims to evaluate the knowledge and views about radiated foods of professors of nutrition working in higher education institutions in the city of Belo Horizonte - MG, Brazil. A total of $86.4 \%$ out of the 66 participants had general knowledge about irradiated foods. However, $71.2 \%$ were not familiar with the process, $75.8 \%$ were totally unaware of the specific legislation, $21.2 \%$ were not sure of the purposes of irradiation, $12.1 \%$ considered irradiated foods radioactive, and $31.8 \%$ believed that food irradiation results in the reduction of the nutritional value of foods. Irradiated foods would not be rejected by professionals with Ph.D degree, but they would be rejected by five masters and six experts questioned. The study concluded that the current higher education of future dietitians and nutritionists has been provided without the minimum necessary knowledge regarding irradiated foods corroborating the negative view of consumers about this kind of food.
\end{abstract}

Keywords: education; food and nutritional security; public health.

\section{Introdução}

A irradiação de alimentos é um método mundialmente reconhecido como efetivo para o controle microbiano em alimentos, sejam estes frescos, na forma de grãos ou mesmo industrializados (ORNELLAS et al., 2006; FURUTA, 1998; LUTTER, 1999), podendo ser aplicado com diferentes propósitos: desinfestação, esterilização, entre outros (OLIVEIRA; SABATO, 2004). As doenças transmitidas por alimentos podem ser minimizadas pelo uso da irradiação de alimentos, a qual reduz significantemente a prevalência de patógenos (LUTTER, 1999; FRENZEN et al., 2001).

O uso da radiação em alimentos está respaldado em argumentos técnicos e na legislação específica. No Brasil, a legislação vigente, promulgada pela Agência Nacional de Vigilância Sanitária (ANVISA), segue as normas internacionais propostas pelo Codex Alimentarius da Organização das Nações Unidas (ONU), pela Food and Agriculture Organization (FAO) e pela International Atomic Energy Agency (IAEA) (BRASIL, 2001).

A resolução no 21 da ANVISA regulamenta o emprego de radiação em alimentos no Brasil, estabelecendo que as fontes de radiação utilizadas devem ser as autorizadas pela Comissão Nacional de Energia Nuclear (CNEN) e que qualquer alimento pode ser irradiado desde que observados os limites mínimos e máximos da dosagem aplicada. A dose mínima deve ser

Recebido para publicação em 8/9/2008

Aceito para publicação em 26/6/2009 (003496)

Departamento de Nutrição Clínica e Social, Escola de Nutrição, Universidade Federal de Ouro Preto - UFOP, Campus Morro do Cruzeiro, s/n, CEP 35400-000,

Ouro Preto - MG, Brasil, E-mail: kesia@enut.ufop.br

${ }^{2}$ Curso de Nutrição, Faculdade de Americana, Rua Joaquim Bôer, 733, CEP 13277-360, Americana - SP, Brasil, E-mail: professoraniurka@hotmail.com

3 Distribuidora de Produtos Hospitalares Ltda. - HMED, Rua Doutor Nogueira Martins, 209, CEP 04143-020, São Paulo - SP, Brasil, CEP 05027-000,

E-mail: eloisa.nascimento@gmail.com

${ }^{*}$ A quem a correspondência deve ser enviada 
suficiente para alcançar a finalidade pretendida; a máxima, inferior àquela que comprometeria as propriedades funcionais e/ou atributos sensoriais do alimento (BRASIL, 2001).

Esta resolução também estabelece que, no painel principal do rótulo dos alimentos irradiados, deve constar a frase "Alimento tratado por processo de irradiação", em letras de tamanho não inferior a um terço do da letra de maior tamanho nos dizeres de rotulagem. Produtos irradiados, utilizados como ingredientes em outro alimento, devem declarar essa circunstância na lista de ingredientes, entre parênteses, após seu nome. Nos alimentos vendidos a granel, é exigida a fixação de faixa ou cartaz com a indicação "produto tratado por irradiação" e/ou com o símbolo da irradiação, a radura (BRASIL, 2001).

A irradiação dos alimentos é realizada em áreas apropriadas (planta estrutural de Cobalto-60), providas de sala de irradiação, piscina de armazenamento, sistema transportador, console de controle e depósito para separar o material irradiado. Os alimentos a serem irradiados são conduzidos ao interior da câmara de irradiação, recebendo a dosagem de radiação gama adequada, a qual foi pré-calculada em função da taxa de dose, isto é, quantidade de radiação por tempo.

O radioisótopo Cobalto-60 é a principal fonte de radiação gama utilizada na irradiação de alimentos devido a sua disponibilidade, custo, apresentar-se na forma metálica e ser insolúvel em água, proporcionando, com isso, maior segurança ambiental (EHLERMANN, 1990). A quantificação das doses de radiação é feita em função da energia absorvida pelo produto irradiado. A unidade de medida utilizada é o Gray (Gy) ou quilogray (kGy), sendo que um Gray equivale a um Joule de energia por quilograma de alimento irradiado. Para aplicação em alimentos, a maioria das doses utilizadas se encontram entre 0,1 e 10,0 kGy (WALDER; CAMARGO, 2007).

Embora existam no Brasil algumas empresas com infraestrutura física, licença para irradiar alimentos e em franca atividade, estudo recente apontou que somente duas indústrias de alimentos no País (Kraft Foods Inc. e Pepsico do Brasil Ltda.) declaram nos rótulos de alguns de seus produtos (salgadinhos de milho, batata frita e biscoito salgado) que estes contêm ingredientes irradiados. Cabe enfatizar que, em 2006, apenas nove produtos foram encontrados no mercado contendo os dizeres exigidos pela legislação vigente (NASCIMENTO; QUINTAES, 2006).

Além de representar cumprimento à legislação nacional e respeito ao consumidor, a importância desta declaração no rótulo também se faz necessária, uma vez que os métodos laboratoriais para determinar se o produto foi tratado por irradiação geralmente são caros, demandam tempo e nem sempre são suficientemente sensíveis, além de serem, evidentemente, inacessíveis ao consumidor final (DELINCÉE, 2002).

Alguns trabalhos educativos, como o uso de vídeos informativos e a realização de feiras culturais, têm conseguido relativo sucesso na transmissão do conhecimento sobre alimentos irradiados ao consumidor, resultando em impacto positivo na aceitação destes (OLIVEIRA; SOBATO, 2004; FOX, 1998). Porém, as organizações médicas, os profissionais da saúde e pesquisadores científicos são as fontes consideradas confiáveis pelos consumidores, quando o assunto versa sobre os alimentos irradiados (HUNTER, 2000).
Entre os profissionais considerados como confiáveis na transmissão de informações relativas aos alimentos irradiados, cabe destacar o nutricionista, por ser o profissional de saúde habilitado para orientar os consumidores sobre a seleção e ingestão de alimentos, comunicando informações sobre os métodos de conservação de alimentos e podendo participar na eliminação de falsas crenças. Adicionalmente, os nutricionistas podem e devem exigir que a legislação vigente sobre alimentos irradiados seja cumprida.

Todavia, nem sempre os nutricionistas têm domínio do assunto. Estudo norte-americano envolvendo o conhecimento de nutricionistas sobre alimentos irradiados e geneticamente modificados mostrou que estes profissionais sabem pouco sobre os alimentos irradiados, sendo o conhecimento deficitário para ambos os alimentos quanto à rotulagem nutricional (WIE; STROHBEHN; SHU, 1998).

No Brasil, até o momento, não há estudos sobre a posição do nutricionista e mesmo de docentes na área da saúde em relação aos alimentos irradiados. Tal situação motivou o presente estudo a avaliar o conhecimento e a atitude frente aos alimentos irradiados entre nutricionistas que atuam na docência de cursos de nutrição em instituições de ensino superior.

\section{Material e métodos}

Foi realizado estudo descritivo envolvendo nutricionistas que atuam como docentes no ensino superior em cursos de nutrição na cidade de Belo Horizonte - MG, Brasil.

O número de instituições de ensino superior que ofertam cursos de graduação em nutrição em Belo Horizonte - MG, Brasil, foi determinado mediante consulta ao Conselho Regional de Nutrição (CRN) da quarta região em agosto de 2006 (CONSELHO..., 2006). No momento da coleta de dados foram encontrados em funcionamento oito cursos de graduação em nutrição, ofertados por oito instituições particulares de ensino superior.

Todos os docentes graduados em nutrição que atuavam nessas instituições foram convidados a participar do estudo, sendo previamente fornecidas a estes explicações sobre o intuito do estudo, a voluntariedade e assegurado o anonimato na apresentação dos dados através de termo de livre consentimento esclarecido. Os critérios de exclusão abrangeram os docentes que estivessem atuando em dois ou mais cursos de nutrição, sendo estes entrevistados uma única vez e, também, aqueles que tivessem além da nutrição, outro curso de graduação.

As informações relativas aos alimentos irradiados foram obtidas por intermédio de questionário estruturado, com 14 questões objetivas, aplicado durante os meses de outubro a dezembro de 2006 por dois estudantes de graduação em nutrição no próprio ambiente de trabalho dos sujeitos da pesquisa.

Considerando a resolução do Ministério da Saúde, 196/1996, o estudo foi previamente avaliado por Comitê de Ética em Pesquisa tendo sua validade científica assegurada (CEP Hospital Heliópolis, processo 400/05). 
Os resultados obtidos foram submetidos à análise por frequência e de variância com aplicação do teste F. Também foi determinado o coeficiente de correlação entre o grau de instrução e o conhecimento sobre alimentos irradiados; e entre a área de atuação e esses saberes. A significância estatística do estudo teve como ponto de corte o valor de $\mathrm{p}<0,05$ (MORETTIN, 2000; STATISTICAL..., 1989).

\section{Resultados e discussão}

No total, 66 docentes nutricionistas foram encontrados ministrando aulas em cursos de graduação em nutrição na cidade de Belo Horizonte - MG, Brasil. Nenhum deles se recusou a participar da pesquisa, o que permitiu que o trabalho fosse realizado com $100 \%$ da população pretendida.

Os docentes entrevistados eram predominantemente do gênero feminino (87,9\%), com idade entre 25 e 55 anos. Cerca de $63,6 \%$ dos professores já tinham feito curso de pós-graduação e $45,5 \%$ referiram ter mais de dez anos de experiência como docente no ensino superior (Tabela 1).

No presente estudo, foi utilizada como premissa para definição de "alimento irradiado" a disposta na legislação vigente, a qual denomina como sendo alimento irradiado todo alimento que tenha sido intencionalmente submetido à ação de radiações ionizantes, com a finalidade de preservá-lo ou para outros fins lícitos, obedecidas as normas elaboradas vigentes (BRASIL, 1973, 2001).

Dos professores participantes, 13,6\% afirmaram desconhecer o que são alimentos irradiados (5 mestres e 4 especialistas). Entre estes, cabe destacar que um dos mestres exerce a atividade de docência na área de alimentos há mais de 10 anos. Estes resultados mostram a importância do processo continuado de aprimoramento de docentes de cursos de nutrição, não somente na área de atuação direta, mas também nas áreas afins.

Tabela 1. Perfil dos docentes de nutrição participantes do estudo sobre conhecimento de alimentos irradiados, Belo Horizonte - MG, 2006.

\begin{tabular}{llrc}
\hline & Característica & $\mathrm{n}$ & $\%$ \\
\hline Gênero & Feminino & 58 & 87,9 \\
& Masculino & 8 & 12,1 \\
Idade & 25 a 35 & 36 & 54,5 \\
(anos) & 36 a 45 & 27 & 41,0 \\
& 46 a 55 & 3 & 4,5 \\
Titulação & Especialistas & 24 & 36,4 \\
& Mestres & 33 & 50,0 \\
Tempo de atuação & Doutores & 9 & 13,6 \\
(anos) & 1 a 5 & 15 & 22,7 \\
& 5 a 10 & 21 & 31,8 \\
Área de atuação & $>10$ & 30 & 45,5 \\
(resposta múltipla) & Clínica & 31 & 47,0 \\
& Saúde pública & 19 & 28,8 \\
& Alimentos & 22 & 33,3 \\
& Outras (esporte, pesquisa, & 1 & 19,7 \\
& estatística, disciplinas básicas, etc.) & & \\
\hline
\end{tabular}

Na cidade de Belo Horizonte, sede do presente estudo, já foi constatado que também os consumidores desconhecem os alimentos irradiados. Pesquisa mostrou que $16 \%$ dos consumidores de Belo Horizonte - MG, Brasil, identificam o termo "alimento irradiado" como tendo significado de "alimento radioativo" (ORNELLAS et al., 2006).

O Joint Expert Committee of Food Irradiation (JECFI), grupo consultor da Organização das Nações Unidas para a Agricultura e Alimentação e da Organização Mundial da Saúde (FAO/OMS), concluiu que um alimento submetido aos raios Gama não se torna radioativo; a irradiação até uma dose média global de 10 kGy não é tóxica, e não traz problemas nutricionais (ORGANIZACIÓN..., 1983).

No entanto, ao ser perguntando aos docentes nutricionistas se os alimentos irradiados são radioativos, $12,1 \%$ dos docentes afirmaram que sim e dois referiram não saber. Foi verificado que $10,5 \%$ dos entrevistados que inicialmente afirmaram saber o que são alimentos irradiados, os classificaram erroneamente como sendo produtos radioativos, assim como $77,8 \%$ dos que referiram não saber o significado de alimentos irradiados.

O equívoco na classificação dos alimentos irradiados como radioativos também foi demonstrado em estudo envolvendo consumidores. Frezen et al. (2001) constaram que 60\% dos consumidores norte-americanos consideram que alimentos irradiados são radioativos.

Na realidade, a irradiação aplicada a alimentos é um processo de "pasteurização" a frio que visa aumentar sua vida de prateleira. Esta técnica pode ser utilizada para destruir insetos, microrganismos patogênicos, fungos e leveduras especialmente em alimentos que são consumidos crus ou parcialmente processados. O retardo de maturação e senescência de frutas e a inibição de brotamento de bulbos e tubérculos também são resultado da sua aplicação. Além de utilizada nos alimentos crus a técnica pode ser aplicada aos alimentos enlatados e congelados (WALDER; CAMARGO, 2007; FARKAS, 1998; ORGANIZACIÓN..., 1995).

Todavia, no presente estudo, foi verificado que uma grande proporção dos professores de nutrição $(71,2 \%)$ desconhece o processo de irradiação de alimentos (Figura 1). Dos 28,8\% que referiram conhecê-lo, cinco são doutores, nove mestres e cinco especialistas.

Pesquisa com consumidores nos Estados Unidos da América revelou que $29 \%$ dos questionados consideram a irradiação um perigo potencial à saúde, comparado com $77 \%$ que identificam as bactérias como um sério perigo e com $66 \%$ que classificam os pesticidas como danosos à saúde humana (BRUHN, 1998). Estas respostas são condizentes com o fato das doenças transmitidas por alimentos permanecerem como sendo uma importante causa de doenças e de morte entre os norte-americanos, deslocando ações intensivas para manter os produtos alimentícios livres de patógenos.

Cabe enfatizar ainda que a mudança genética de agentes patogênicos induzida pela irradiação nos alimentos não produz riscos à saúde, não havendo diferença qualitativa entre o tipo de mudança provocada pela irradiação ionizante e a induzida 
por qualquer outro método de pasteurização ou a conservação parcial (ORGANIZACIÓN..., 1995). Os consumidores podem reduzir os riscos de contrair doenças veiculadas por alimentos ao adquirirem produtos alimentícios tratados por radiação, como as carnes (FRENZEN et al., 2001).

Hunter (2000) relatou que a segurança alimentar, o controle de infestações e a maior vida de prateleira dos produtos são as principais razões motivadoras da compra dos alimentos irradiados pelos consumidores. Por outro lado, tem sido verificado que a ausência ou presença de informação sobre os alimentos irradiados determina sua aceitação pelo consumidor (ORNELLAS et al., 2006; BORD; O’CONNOR, 1989).

No presente estudo, a maior parte dos docentes entrevistados $(78,8 \%)$ afirmou que conhece alguma das finalidades do processo de irradiação de alimentos. Curiosamente, dois dos nove docentes, que não sabiam o que são alimentos irradiados, afirmaram saber alguns dos objetivos da aplicação da irradiação a gêneros alimentícios. Já entre os 21,2\% que desconhecem as finalidades da irradiação de alimentos, um era doutor, nove mestres e os demais especialistas.

Com respeito ao consumo dos alimentos irradiados, $21,2 \%$ dos docentes referiram ter certeza de já terem ingerido alimentos irradiados, 15,2\% negaram e 63,6\% não souberam dizer se consumiram ou não. $\mathrm{O}$ alto percentual de dúvida pode estar associado ao fato de raramente estar declarado junto aos alimentos comercializados no Brasil se este ou algum de seus

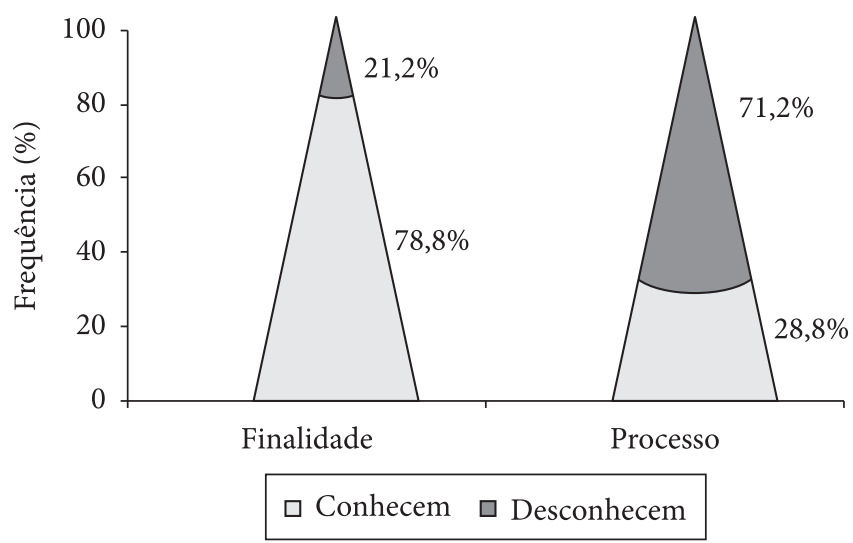

Figura 1. Conhecimento relativo à finalidade e ao processo utilizado na irradiação de alimentos pelos docentes participantes do estudo, Belo Horizonte - MG, 2006. ingredientes foi submetido à irradiação (NASCIMENTO; QUINTAES, 2006).

O desconhecimento sobre o processo de irradiação e seus efeitos pode ser considerado como o principal motivo de rejeição ao consumo de alimentos irradiados. No entanto, $80,3 \%$ dos professores manifestaram que consumiriam esses alimentos, 3\% ficaram em dúvida e 16,7\% afirmaram que não os consumiriam, caso se deparassem com um alimento declaradamente irradiado.

Comparando a titulação com a possibilidade de consumo de alimentos irradiados, estes não seriam recusados pelos doutores, enquanto $12 \%$ dos mestres e $25 \%$ dos especialistas os rejeitariam, no entanto, não foi encontrada relação estatisticamente significativa entre estas variáveis (Tabela 2 ).

Os resultados obtidos no presente estudo são condizentes com o trabalho realizado por Oliveira e Sobato (2004), no qual o grau de interesse na aquisição de alimentos irradiados foi positivamente associado ao maior nível educacional dos congressistas presentes no International Nuclear Atlantic Conference e na XV Reunião Anual do Instituto Biológico, realizados no ano de 2002 no Rio de Janeiro - RJ, Brasil, e em São Paulo - SP, Brasil, respectivamente.

Com respeito ao valor nutricional dos alimentos irradiados, é sabido que a irradiação promove poucas alterações no teor dos nutrientes. O teor das vitaminas tiamina e riboflavina podem ser ligeiramente reduzidos nos alimentos tratados por radiação gama (VILLAVICENCIO et al., 2000), enquanto que a o ácido ascórbico tem sua forma química ativa diminuída em função da dose da radiação gama aplicada (IEMMA et al., 1999). Mesmo assim, cerca de um terço dos docentes entrevistados $(31,8 \%)$ considerou que a irradiação de alimentos resulta em impacto negativo no valor nutritivo dos alimentos e $4,5 \%$ não sabem. Em estudo com nutricionistas norte-americanos, foi verificado que 25,4\% também acreditavam erroneamente que este método reduz o valor nutricional dos alimentos (WIE; STROHBEHN; SHU, 1998).

No presente estudo, foi contraditoriamente notado que $14,3 \%$ dos docentes que acreditam que a irradiação reduz o valor nutritivo dos alimentos, anteriormente tinha afirmado desconhecer o que são alimentos irradiados. Entre os 21 participantes que referiram desconhecer o que são alimentos irradiados, $29,17 \%$ são doutores, $33,33 \%$ são mestres e $37,5 \%$ são especialistas.

Tabela 2. Correlação de variáveis pesquisadas sobre conhecimentos de alimentos irradiados com nutricionistas docentes em cursos de Nutrição.

\begin{tabular}{|c|c|c|c|c|}
\hline \multirow[t]{2}{*}{ Pergunta } & \multicolumn{2}{|c|}{ Grau de instrução } & \multicolumn{2}{|c|}{ Área de atuação } \\
\hline & $\mathrm{r}$ & $\mathrm{p}$ & $\mathrm{r}$ & $\mathrm{p}$ \\
\hline Alimentos irradiados são radiativos? & $-0,1617$ & $\mathrm{p}=0,0794$ & 0,1293 & $\mathrm{p}=0,0911$ \\
\hline Objetivo da irradiação de alimentos? & $-0,1500$ & $\mathrm{p}=0,1682$ & $-0,0171$ & $\mathrm{p}=0,0685$ \\
\hline Qual a substância utilizada em irradiação de alimentos? & $-0,2495$ & $\mathrm{p}=0,4166$ & 0,0792 & $\mathrm{p}=0,1267$ \\
\hline Consumiria alimentos irradiados? & $-0,1564$ & $\mathrm{p}=0,0698$ & 0,0121 & $\mathrm{p}=0,0847$ \\
\hline A irradiação altera o valor nutricional dos alimentos? & 0,0797 & $\mathrm{p}=0,1621$ & $-0,0492$ & $\mathrm{p}=0,1068$ \\
\hline A irradiação traz benefícios à saúde? & 0,2540 & $\mathrm{p}=0,0776$ & 0,1573 & $\mathrm{p}=0,3188$ \\
\hline
\end{tabular}


Cabe enfatizar que os métodos de conservação de alimentos existentes podem interferir em maior ou menor grau na composição dos produtos e também nos caracteres sensoriais. A radiação gama $(1,0 \mathrm{kGy})$ aplicada em aipo e repolho minimamente processados não alterou os atributos sensoriais, reduzindo significativamente a contaminação microbiana (LÓPEZ et al., 2005). A beterraba vermelha minimamente processada, embalada e submetida a diferentes doses de radiação gama $(1,0$; e $1,5 \mathrm{kGy})$ manteve seu padrão de aceitabilidade por 20 dias (HERNANDES et al., 2007).

No aspecto relativo ao valor nutricional, a conservação de alimentos por irradiação tem baixo impacto, se comparada com as demais tecnologias existentes (WIE; STROHBEHN; SHU, 1998), com exceção de certos nutrientes específicos como o tocoferol e os ácidos graxos (MALISKA, 2000; BRITO; VILLACICENCIO; MANCINI-FILHO, 2002). Dependendo do alimento e da dose de radiação usada, algumas características sensoriais podem se mostrar alteradas (OLIVEIRA; SOBATO, 2004).

Os resultados de pesquisas realizadas nas últimas quatro décadas não mostraram problemas de saúde relacionados ao consumo de alimentos irradiados. Estudos com animais de experimentação (toxicidade aguda e crônica, carcinogênese, teratogênese e mutagenicidade) e com seres humanos (astronautas, tropas, voluntários) têm sido conduzidos sem evidências de efeitos nocivos derivados desse consumo (YU et al., 2004; DONALD; ENGEL, 1993). Em diversos países, pacientes imunodeprimidos têm indicação para consumo de alimentos esterilizados por radiações ionizantes (BUTTERWECK, 1995).

No presente estudo, 40,9\% dos docentes consideraram que o consumo de alimentos irradiados poderia ter impacto benéfico na saúde do consumidor. Por outro lado, 53\% refutaram este benefício e 6,1\% não souberam responder. Estes resultados são similares aos obtidos com consumidores leigos por Ornellas et al. (2006), para quem, 62\% dos participantes responderam não saber se a irradiação de alimentos pode trazer danos à saúde do consumidor.

Entre os que afirmaram que os alimentos irradiados são benéficos à saúde, $66,7 \%$ são doutores, $42,4 \%$ mestres e $28,2 \%$ especialistas, mas não houve relação estatisticamente significativa (Tabela 2), entre o grau de instrução do docente e seus conhecimentos sobre o impacto dos irradiados nos humanos.

Os docentes entrevistados no estudo foram praticamente unânimes (98,5\%) com relação à necessidade de estampar nos rótulos se os alimentos são irradiados ou formulados com ingredientes irradiados. No entanto, foi observado que 75,8\% desconhecem a legislação específica. Os 16 profissionais que referiram conhecer esta legislação, mencionaram a ANVISA, seguida pelo Codex Alimentarius e pela OMS como sendo as fontes destas regulamentações. Alguns docentes afirmaram conhecer mais de uma legislação simultaneamente.
Oliveira e Sobato (2004), entrevistando congressistas do International Nuclear Atlantitc Conference, constataram que $40 \%$ dos declarantes desconheciam a legislação sobre alimentos irradiados no Brasil. Estudo norte-americano, envolvendo o conhecimento de nutricionistas sobre alimentos irradiados e geneticamente modificados, mostrou que estes profissionais sabem menos sobre os alimentos irradiados do que sobre os geneticamente modificados, sendo que, no quesito da rotulagem nutricional, o conhecimento se mostrou deficitário para ambos os produtos (WIE; STROHBEHN; SHU, 1998).

No Brasil, o profissional nutricionista é habilitado para atuar em certas áreas de conhecimento, segundo o Conselho Federal de Nutrição (CONSELHO..., 2006). Não houve relação estatisticamente significativa (Tabela 2) entre a área de atuação do docente e os aspectos questionados nesta pesquisa. No entanto, chamou a atenção o fato de nutricionistas que ministram disciplinas na área de alimentos (Exemplos: Composição de alimentos, Bromatologia, Tecnologia de alimentos e Higiene de alimentos) desconheçam conceitos essenciais relativos aos alimentos irradiados. Nas áreas de clínica e de saúde pública, também esta constatação é dramática, uma vez que o tema é atual e guarda estreita relação com o consumo e a segurança alimentar como premissa para a manutenção e/ ou restabelecimento da saúde humana (Figura 2).

O estudo propiciou outra verificação importante ao constatar que $97 \%$ dos docentes não abordam, direta ou indiretamente, nenhum tópico sobre alimentos irradiados em suas disciplinas. Foi constado que somente dois (3\%) dos docentes da área de alimentos abordam os alimentos irradiados em suas disciplinas. Estes docentes anteriormente se mostraram favoráveis à rotulagem nutricional dos alimentos irradiados e sem rejeição quanto ao seu consumo, referindo saber que o processo de irradiação não modifica o valor nutricional dos alimentos.

Embora aparentemente o tema alimentos irradiados possa parecer ser peculiar à área de alimentos, são diversos os assuntos dentro das três áreas básicas de conhecimento da ciência

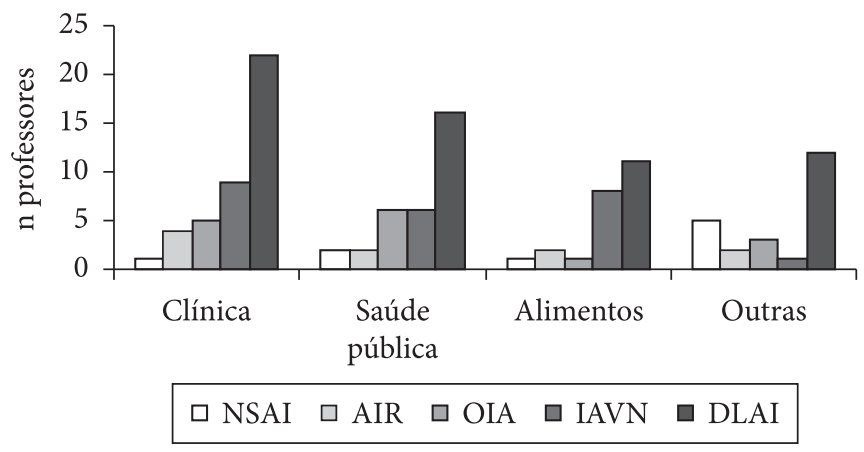

Figura 2. Distribuição por área de atuação dos nutricionistas que trabalham como professores em cursos de nutrição que: NSAI: não sabem o que são alimentos irradiados; AIR: consideram que alimentos irradiados são radioativos; OIA: não sabem os objetivos da irradiação de alimentos; IAVN: acreditam que a irradiação afeta o valor nutritivo do alimento; e DLAI: desconhecem a legislação sobre alimentos irradiados. 
Quadro 1. Assuntos relacionados aos alimentos irradiados nas diferentes áreas do conhecimento.

\begin{tabular}{|cl|}
\hline Área de conhecimento & \multicolumn{1}{c|}{ Tópico } \\
\hline Alimentos & Rotulagem dos alimentos \\
& Utilização da irradiação no controle de micro-organismos deterioradores e patogênicos em alimentos \\
& Métodos de conservação dos alimentos \\
& Aquisição e conservação de alimentos irradiados \\
& Valor nutricional dos alimentos irradiados \\
& Vida de prateleira de alimentos submetidos a distintas técnicas de conservação \\
& Análise sensorial de alimentos \\
& Benefícios da irradiação em embalagens para dietas enterais e parenterais \\
& Alimentos irradiados para pacientes imunodeprimidos, etc. \\
& Radapertização de frascos e equipamentos médicos e hemocentros \\
& Uso dos alimentos irradiados como medida de prevenção de doenças transmitidas pelos alimentos \\
& Custo dos alimentos irradiados, valor nutricional e consumo alimentar populacional \\
\hline
\end{tabular}

da nutrição nas quais os alimentos irradiados poderiam ser abordados pelos docentes. Com a finalidade de tornar aplicação do tema mais próxima e acessível, foi elaborada uma proposta elementar ilustrativa (Quadro 1).

\section{Conclusões}

Todos os nutricionistas que ministram aulas em cursos de graduação em nutrição na cidade de Belo Horizonte MG, Brasil, participaram do estudo e apresentaram baixo conhecimento sobre alimentos irradiados. Apesar de a maioria referir saber o significado do termo, contradições foram evidenciadas, indicando deficiência no saber dos conceitos básicos da técnica, de seus possíveis usos, de seus impactos na saúde humana e da legislação vigente relacionada.

Há desconhecimento dos docentes quanto à ocorrência de consumo de alimentos irradiados devido à não indicação em rótulos. No entanto, muitos afirmaram ( $80 \%)$ que consumiriam alimentos irradiados, caso tivessem conhecimento a respeito, ou seja, admitindo que seu conhecimento sobre o assunto é limitado.

Os resultados demonstram que a formação dos futuros nutricionistas pode estar sendo realizada sem o mínimo de conhecimento necessário a respeito dos alimentos irradiados. Tal fato é preocupante, já que o profissional da nutrição tem um papel fundamental na transmissão de informações à população sobre formas e benefícios dos métodos de conservação de alimentos e eliminar falsas crenças, assim como colaborar para que a legislação nacional vigente sobre alimentos irradiados seja cumprida.

A introdução de maior e melhor conhecimento técnico sobre alimentos irradiados, mediada por professores que efetivamente dominam o tema, poderá contribuir para a formação de nutricionistas devidamente habilitados em todas as esferas de atuação definidas pelo Conselho Federal de Nutrição no Brasil.

\section{Referências bibliográficas}

BORD, R. J.; O'CONNOR, R. E. Who wants irradiated food? Untangling complex public opinion. Food Technology, v. 43, n. 10, p. 87-90, 1989.
BRASIL. Agência Nacional de Vigilância Sanitária - ANVISA.

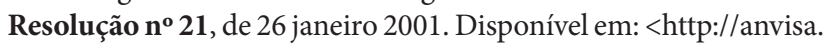
gov.Br/legis/resol/21_01rdc.htm>. Acesso em: 11 nov. 2006.

BRASIL. Decreto n. 72.718, de 29 de agosto de 1973. Normas gerais sobre irradiação de alimentos. Diário Oficial da República Federativa do Brasil, Brasília, 30 de agosto de 1973.

BRITO, M. S.; VILLACICENCIO, A. L. C. H.; MANCINI-FILHO, J. Effects of irradiation on trans fatty acids formation in ground beef. Radiation Physics and Chemistry, v. 63, n. 3-6, p. 337-340, 2002.

BRUHN, C. M. Consumer acceptance of irradiated food: theory and reality. Radiation Physics and Chemistry, v. 52, n. 1-6, p. 129-133, 1998.

BUTTERWECK, J. S. Sterile diets for the immuno-compromised: Is there a need? Radiation Physics and Chemistry, v. 46, n. 4-6, p. 601-604, 1995.

CONSELHO FEDERAL DE NUTRIÇÃO. Formação profissional. Disponível em: <http://www.cfn.org.br>. Acesso em: 02 ago. 2006.

DELINCÉE, H. Analytical methods to identify irradiated food - a review. Radiation Physics and Chemistry, v. 63, n. 3-6, p. 455-458, 2002.

DONALD, D. D.; ENGEL, E. R. Status of food irradiation in the United States. Radiation Physics and Chemistry, v. 42, p. 1-3, p. 289-296, 1993.

EHLERMANN, D. A. E. Food Irradiation. In: SPIESS, W. E. L.; SCHUBERT, H. (Ed.). Engineering and food: Preservation processes and related techniques. London: Elsevier Applied Science, 1990. p. 760-73.

FARKAS, J. Irradiation as a method for decontamination food - a review. International Journal of Food Microbiology, v. 44, n. 3, p. 189-204, 1998.

FOX, A. J. Market trials of irradiated chicken. Radiation Physics and Chemistry, v. 52, n. 6-12, p. 63-66, 1998.

FRENZEN, P. D. et al. Consumer acceptance of irradiated meat and poultry in the United States. Journal of Food Protection, v. 64, n. 12, p. 2020-2026, 2001.

FURUTA, M. Current status of information transfer activity on food irradiation and consumer attitudes in Japan. Radiation Physics and Chemistry, v. 71, n. 1-2, p. 499-502, 1998.

HERNANDES, N. K. et al. Testes sensoriais de aceitação da beterraba vermelha (Beta vulgaris ssp. vulgaris L.), cv. Early Wonder, minimamente processada e irradiada. Ciência e Tecnologia de Alimentos, v. 27, suppl. 1, p. 64-68, 2007. 
HUNTER, C. Changing attitudes to irradiation throughout the food chain. Radiation Physics and Chemistry, v. 57, n. 3-6, p. 239-243, 2000.

IEMMA, J. et al. Radiação gama na conservação do suco natural de laranja. Scientia Agricola, v. 56, n. 4, p. 1193-1198, 1999.

LÓPEZ, L. etal. Effect of gamma irradiation on the microbiological quality of minimally processed vegetables. Archivos Latinoamericanos de Nutrición, v. 55, n. 3, p. 287-292, 2005.

LUTTER, R. Food irradiation - the neglected solution to foodborne illness. Science, v. 286, n. 5448, p. 2275-2276, 1999.

MALISKA, C. Conservação de alimentos por irradiação. Higiene Alimentar, v. 14, n. 68, p. 69-70, 2000.

MORETTIN, L. G. Estatística básica. Inferência. São Paulo: Makron Books, 2000.

NASCIMENTO, E. S.; QUINTAES, K. D. Produção e comercialização de alimentos irradiados no Brasil. Nutrição Brasil, v. 5, n. 2, p. 99-104, 2006.

OLIVEIRA, I. B.; SOBATO, S. F. Dissemination of the food irradiation process on different opportunities in Brazil. Radiation Physics and Chemistry, v. 71, n. 1-2, p. 493-497, 2004.

ORGANIZACIÓN DE LAS NACIONES UNIDAS PARA LA AGRICULTURA Y LA ALIMENTACIÓN / ORGANIZACION MUNDIAL DE LA SALUD. Informe del 150 periodo de sesiones de la comisión mixta FAO/OMS del Codex Alimentarius, 1983.
Disponível em: <http://www.fao.org/docrep/meeting/ 005/AC331S/ AC331S02.htm>. Acesso em: 30 set. 2006.

ORGANIZACIÓN MUNDIAL DE LA SALUD (OMS). Inocuidad e idoneidad nutricional de los alimentos irradiados. Genebra, 1995.

ORNELLAS, C. B. D. et al. Atitude do consumidor frente à irradiação de alimentos. Ciência e Tecnologia de Alimentos, v. 26, n. 1, p. 211-213, 2006.

STATISTICAL ANALYSIS SYSTEM - SAS. User's guide: statistics. 6 ed. Cary, 1989.

VILLAVICENCIO, A. L. C. H. et al. Effect of gamma irradiation on the thiamine, riboflavin and vitamin B6 content in two varieties of Brazilian beans. Radiation Physics and Chemistry, v. 57, n. 3-6, p. 299-303, 2000 .

WALDER, J. M. M.; CAMARGO, A. C. Irradiação de alimentos. Divulgação da tecnologia de irradiação de alimentos e outros materiais. 2007. Disponível em: <http://www.cena.usp.br/ irradiacao/irrad_alim.htm>. Acesso em: 8 ago. 2007.

WIE, S. H.; STROHBEHN, C. H.; SHU, C. H. C. Iowa dietitians` attitudes toward and knowledge of genetically engineered and irradiated foods. Journal of the American Dietetic Association, v. 98 , n. 11, p. 1331-1333, 1998.

YU, Y. B. et al. Toxicological safety and stability of the components of an irradiated Korean medicinal herb, Paeoniae Radix. Radiation Physics and Chemistry, v. 71, n. 1-2, p. 117-121, 2004. 